\title{
TRATAMIENTO FARMACOLÓGICO DEL TRASTORNO BIPOLAR EN EL EMBARAZO
}

\section{PHARMACOLOGICAL TREATMENT OF BIPOLAR DISORDER DURING PREGNANCY}

\section{Hugo RODRÍGUEZ}

${ }^{1}$ Auxiliar de la Enseñanza de Psiquiatría, Facultad de Ciencias Médicas, Universidad Nacional de Asunción, San Lorenzo - Paraguay.

Cómo citar este artículo: Rodríguez H. Tratamiento farmacológico del trastorno bipolar en el embarazo. Medicina Clínica y Social. 2017;1(1):42-48.

\section{RESUMEN}

El tratamiento farmacológico del trastorno bipolar en una mujer gestante constituye una situación compleja a la que nos enfrentamos todos los profesionales de salud mental. Representa una instancia que suele despertar temor tanto en la usuaria y su entorno, como en los agentes de salud, y esto frecuentemente dificulta la consecución del objetivo, es decir, efectuar las decisiones terapéuticas más convenientes para la usuaria y el embrión o feto, haciendo partícipe de una manera informada a todos los protagonistas. Si bien uno de los objetivos principales es minimizar la exposición del embrión o feto a fármacos con riesgo teratogénico, no por eso deben indicarse dosis subterapéuticas a la madre, ya que con esa conducta se estaría exponiendo al feto a un doble riesgo: el trastorno psiquiátrico no tratado y el potencial teratogénico del medicamento. Ante la complejidad que implica el tratamiento del trastorno bipolar durante el embarazo, en este artículo de revisión se repasan diversas estrategias que puedan ser adoptadas según las condiciones específicas de cada situación.

Palabras clave: Tratamiento farmacológico; Embarazo; Trastorno bipolar.

\section{ABSTRACT}

The pharmacological treatment of bipolar disorder in a pregnant woman is a complex situation faced by all mental health professionals. It represents an instance that usually arouses fear in the user and its environment, as well as in the health agents, and this frequently hinders the achievement of the objective, that is, to make the more convenient therapeutic decisions for the user and the embryo or fetus, informing all the protagonists in an informed way. Although one of the main objectives is to minimize the exposure of the embryo or fetus to drugs with teratogenic risk, sub therapeutic doses should not be indicated to the mother, since this behavior would expose the fetus to a double risk: the mother's psychiatric disorder and the teratogenic potential of the drug. Given the complexity involved in the treatment of bipolar disorder during pregnancy, this review article reviews various strategies that can be adopted according to the specific conditions of each situation.

Keywords: Pharmacological treatment; Pregnancy; Bipolar disorder. 


\section{INTRODUCCIÓN}

El tratamiento del trastorno bipolar en una mujer gestante constituye una situación compleja a la que nos enfrentamos todos los profesionales de salud mental. Representa una instancia que suele despertar temor tanto en la usuaria y su entorno, como en los agentes de salud, y esto frecuentemente dificulta la consecución del objetivo, es decir, efectuar las decisiones terapéuticas más convenientes para la usuaria y el embrión o feto, haciendo partícipe de una manera informada a todos los protagonistas.

El tratamiento farmacológico del trastorno bipolar durante el período de gravidez tropieza con un importante inconveniente. Los datos que se poseen actualmente acerca de la seguridad reproductiva de los medicamentos empleados para el efecto, provienen de estudios retrospectivos o reportes de casos clínicos, ya que los estudios controlados aleatorizados resultan éticamente inviables.

Es necesario adoptar actitudes que dirijan hacia un empleo racional de los recursos terapéuticos, y evitar la simplificación de la situación asumiendo que "siempre será preferible evitar la utilización de psicofármacos". Los riesgos obstétricos derivados de un trastorno psiquiátrico no tratado pueden ser tanto o más importantes que aquellos derivados, por ejemplo, del potencial teratogénico de los fármacos (1).

Si bien uno de los objetivos principales es minimizar la exposición del embrión o feto a fármacos con riesgo teratogénico, no por eso deben indicarse dosis subterapéuticas a la madre, ya que con esa conducta se estaría exponiendo al feto a un doble riesgo: el trastorno psiquiátrico no tratado y el potencial teratogénico del medicamento (2).

La incidencia de los trastornos psiquiátricos durante la gestación es similar a la registrada en otros períodos de la vida, pero la prevalencia de síntomas afectivos y cognitivos subsindrómicos en este período es particularmente alta (3).

\section{CONSENTIMIENTO INFORMADO}

La prescripción de un tratamiento farmacológico a una mujer gestante requiere la obtención de un consentimiento informado. En ocasiones, debido por ejemplo a una intención de tranquilizar o evitar preocupaciones a la usuaria y su entorno, se cae en el error de no brindar la información completa sobre los riesgos y beneficios de cada opción terapéutica y de la opción de negarse al tratamiento. Esto debe ser evitado, a través de una interacción abierta y fluida entre el profesional (o los profesionales) y la mujer y su entorno (siempre que resulte posible, debe también obtenerse el consentimiento informado del otro futuro progenitor). El profesional debe obtener, en cada caso, la información más completa y actualizada posible sobre la seguridad reproductiva de los fármacos que se plantean como opciones, sin olvidar las limitaciones de estos datos debidas a las dificultades metodológicas para obtenerlos. Puede resultar útil recurrir a los sistemas de clasificación de los fármacos según su seguridad reproductiva (por ejemplo, el sistema $A B C$ de la Food And Drug Administration), teniendo presente que son herramientas orientativas, y no deben constituir la principal (y mucho menos única) fuente de información para la toma de decisiones $(2,4,5)$. 


\section{TRASTORNO BIPOLAR DURANTE EL EMBARAZO}

El trastorno bipolar muestra un curso variable durante la gestación (3). Se considera que, sin tratamiento, este trastorno muestra una tasa de recurrencia del 32 al 67\% durante el embarazo. El papel protector contra el trastorno bipolar que se atribuía al embarazo en el pasado, hoy se considera infundado (6).

Viguera y colaboradores (7) sostienen que los factores asociados con recurrencia de la enfermedad durante la gestación son (8):

a. más de cinco años de diagnóstico del trastorno;

b. inicio a una edad más temprana;

c. diagnóstico de trastorno bipolar tipo II;

d. antecedente de ciclado rápido;

e. estabilidad clínica desde el último episodio por un período corto antes de la gestación;

f. más de un episodio anterior al año;

g. antecedente de intento de suicidio; $y$,

h. comorbilidad psiquiátrica.

El embarazo solo se considerará un factor de riesgo asociado si no es planeado. Los factores de riesgo relacionados con el tratamiento son (8):

a. polifarmacia con más de dos psicotrópicos;

b. empleo de antidepresivos;

c. estabilizador del ánimo primario distinto del litio;

d. viraje durante el tratamiento con un antidepresivo; $y$,

e. suspensión abrupta del estabilizador del ánimo (en menos de 2 semanas).

Los riesgos asociados a la manía no tratada en una paciente gestante son (4, 9):

- Mayor riesgo de enfermedades de transmisión sexual debido a conducta sexual promiscua.

- Incremento del riesgo de exposición a situaciones de violencia u otros estresantes (pérdida de vivienda, pérdida laboral).

- Aumento del riesgo de consumo de sustancias.

- Disminución de cuidados prenatales.

- Incremento del riesgo de internaciones prolongadas.

- Mayor incidencia de parto prematuro.

- Aumento del riesgo de psicosis puerperal.

\section{CONSIDERACIONES GENERALES SOBRE EL TRATAMIENTO FARMACOLÓGICO}

En relación al tratamiento farmacológico del trastorno bipolar en el embarazo, se deben tener en cuenta inicialmente datos de la historia clínica de la mujer, de manera a realizar una adecuada elección de los recursos terapéuticos. Estos datos son: a) respuesta previa a psicofármacos, b) severidad del trastorno, c) duración del período eutímico con y sin medicación, d) tiempo que tarda en aparecer la recaída luego de interrumpir la medicación y e) tiempo que tarda la paciente en recuperarse una vez reanudada la farmacoterapia (10). 
Los tres estabilizadores del ánimo más utilizados (litio, ácido valproico y carbamazepina) incrementan el riesgo teratogénico, por lo tanto, su empleo debe evitarse durante el primer trimestre de la gestación siempre que sea posible.

La exposición a un fármaco con riesgo teratogénico puede producir sus efectos según la edad gestacional en que se produce la exposición: el desarrollo y cierre del tubo neural puede afectarse hasta los 32 días luego de la concepción; el corazón puede sufrir alteraciones entre los días 21 y 56 después de la concepción; la formación de los labios y el paladar puede verse afectada entre los días 42 y 63. Las malformaciones craneofaciales pueden generarse inclusive pasado el primer trimestre, así como las alteraciones del desarrollo neuroconductual, que se constatarán luego del nacimiento (9).

El mayor riesgo de teratogénesis se registra durante el primer trimestre del embarazo. No obstante, la exposición posterior puede asociarse a otros riesgos perinatales, como son: riesgo de malformaciones menores, efectos conductuales, parto pretérmino y bajo peso al nacer (10).

En una mujer que recibe tratamiento farmacológico para el trastorno bipolar, resulta ideal que el embarazo sea planeado, a fin de evitar una exposición inadvertida del embrión o feto a psicofármacos riesgosos.

Cuando la mujer presenta un trastorno de curso benigno y está atravesando un período asintomático prolongado, se puede proceder a la suspensión gradual de los estabilizadores del ánimo, previamente a la concepción $(4,11)$.

Es deseable realizar, previamente a la concepción, una prueba de suspensión gradual del tratamiento farmacológico, la cual, si resulta exitosa, otorga mayor confianza para enfrentar la ausencia de farmacoterapia durante los primeros tres meses de gestación $(10,12,13)$.

En los casos en los que la confirmación de la gestación se da luego de haber transcurrido el período de mayor riesgo teratogénico, la interrupción del tratamiento farmacológico puede representar un riesgo para la mujer y el feto y brindar beneficios mínimos. En dichos casos se debe realizar un ajuste individualizado del plan de tratamiento, teniendo en cuenta la estabilidad de la paciente, la edad gestacional, el psicofármaco en cuestión y las preferencias de la paciente (y su pareja, cuando corresponda) $(10,14)$.

Las mujeres con antecedentes de recurrencias frecuentes, hospitalizaciones previas o intentos de autoeliminación, constituyen una población de alto riesgo de recaída y resistencia al tratamiento; por lo tanto, en estos casos, la continuación del tratamiento farmacológico suele representar la estrategia más prudente (8). Dentro de este grupo, puede haber usuarias que opten por interrumpir el tratamiento farmacológico antes de la concepción; con este fin puede procederse a mantener el tratamiento farmacológico hasta que se constate fehacientemente el estado de gravidez, y a partir de ahí suspender gradualmente la medicación. El riesgo de exposición embrionaria que reviste esta estrategia es mínimo, ya que la circulación útero-placentaria se establece aproximadamente dos semanas después de la concepción. Esta estrategia resulta particularmente útil en mujeres de edad avanzada para el embarazo, las cuales suelen requerir de un tiempo mayor para concebir. La desventaja que implica este método radica en la suspensión relativamente brusca del tratamiento farmacológico, que conlleva un riesgo de recaída incrementado $(4,11,12)$. 
Cuando se optó por la interrupción de la farmacoterapia durante el primer trimestre, la decisión sobre la reanudación o no del tratamiento medicamentoso debe realizarse con un juicio clínico adecuado. En algunos casos se elige esperar la reaparición de sintomatología para reiniciar la medicación, mientras que otra alternativa es reanudar la medicación luego del primer trimestre, minimizando el riesgo de recurrencia (15).

No debe olvidarse que existen alternativas al tratamiento con estabilizadores del ánimo. Dentro de las opciones farmacológicas se debe tener presentes a los antipsicóticos típicos y atípicos, que, como grupo, se consideran fármacos con un menor riesgo teratogénico que los anticonvulsivantes (si bien debe analizarse individualmente cada opción farmacológica y con información actualizada). También debe considerarse a la terapia electroconvulsiva, que constituye una opción segura y eficaz (15).

Las mujeres que eligen cambiar la medicación por un antipsicótico típico o uno atípico asociado a hiperprolactinemia, podrían ver afectada su capacidad de concepción, tardando más o disminuyendo la posibilidad de conseguirla $(9,10)$.

\section{TRATAMIENTO DE UN EPISODIO AGUDO DURANTE LA GESTACIÓN}

Las conductas terapéuticas para el tratamiento de fase aguda de los posibles episodios de un trastorno bipolar durante el embarazo, pueden resumirse como sigue $(14,16)$ :

\section{Manía aguda}

- Si la mujer no recibe medicación: Considerar un antipsicótico atípico o típico, emplear la mínima dosis eficaz posible.

- Si la mujer está recibiendo tratamiento de mantenimiento: Comprobar cumplimiento correcto del tratamiento.

Si está recibiendo un antipsicótico, incrementar la dosis; o considerar el cambio a un antipsicótico, si no lo está recibiendo.

Si no hay respuesta y el cuadro es grave, considerar terapia electroconvulsiva, litio y, excepcionalmente, valproato.

- Si no hay alternativa razonable al empleo de valproato:

Usar la menor dosis posible y considerar la potenciación con medicación adicional, evitando la carbamazepina. En este sentido, tener presente que la polifarmacia, en especial la combinación de valproato y lamotrigina, incrementa el riesgo teratogénico.

La dosis máxima aconsejable sería de $1000 \mathrm{mg} /$ día, en dosis divididas y en forma de liberación prolongada. Se debe suplementar con ácido fólico ( $5 \mathrm{mg} / \mathrm{día})$.

\section{Episodio depresivo}

- Ante un cuadro leve:

Plantear primero opciones psicoterapéuticas: terapia cognitivo-conductual, intervenciones psicológicas breves, autoayuda guiada.

Si no hay mejoría, recurrir a antidepresivos.

- Si se trata de un cuadro moderado o grave:

Considerar la terapia cognitivo-conductual en cuadros moderados.

Considerar farmacoterapia combinada con intervenciones psicológicas estructuradas. 
Considerar quetiapina sola o combinada con inhibidores selectivos de la recaptación de serotonina, monitorizar la posibilidad de viraje.

Considerar terapia electroconvulsiva, particularmente durante el primer trimestre.

\section{CONCLUSIONES}

Ante la complejidad que implica el tratamiento del trastorno bipolar durante el período fértil en la mujer, se repasaron diversas estrategias que pueden ser adoptadas según las condiciones específicas de cada situación.

Resulta evidente la conveniencia de evitar ciertas actitudes o procedimientos que terminan complicando la evolución o aumentando los riesgos, como, por ejemplo, la utilización de dosis por debajo de los rangos terapéuticos, o la suposición de que siempre resulta más conveniente la interrupción de la farmacoterapia durante la gestación. En relación a esto último, se tienen abundantes datos acerca de lo perjudicial que puede ser un episodio afectivo para la evolución misma del embarazo.

Una recomendación que no se puede soslayar es la de obtener la mayor cantidad posible de información actualizada sobre cada una de las opciones terapéuticas que planteamos a una usuaria (y su pareja, cuando corresponda), ya que los datos sobre la seguridad reproductiva de los psicofármacos se renuevan y modifican periódicamente, con las dificultades metodológicas ya mencionadas que enfrenta esta área de investigación.

Otro procedimiento fundamental es el de la obtención del consentimiento informado, el cual no debe ser asumido como una herramienta para deslindar responsabilidades. Este proceso implica compartir con la paciente y su entorno toda la información completa y actualizada descrita más arriba; de manera que, tanto usuarios como profesionales, participen de la toma de decisiones en el tratamiento. De esta manera, es común que se mitigue ostensiblemente la angustia generada por situaciones cargadas de incertidumbre.

Teniendo en cuenta todas las recomendaciones descritas, es probable que las decisiones terapéuticas, ya sea que impliquen suspensión, cambio o mantenimiento de algún recurso terapéutico, se tomen con un mayor nivel de seguridad y esto redunde en mejores resultados para la mujer y su entorno cercano.

\section{CONFLICTOS DE INTERÉS Y FUENTE DE FINANCIACIÓN}

El autor declara no poseer conflictos de interés. Fuente de financiación: ninguna.

\section{REFERENCIAS BIBLIOGRÁFICAS}

1. Franco C, Goikolea JM, García-Esteve L, Imaz ML, Benabarre A, Vieta E. Tratamiento de los trastornos mentales en la mujer embarazada. Jano Extra. 2008; 1(714): 55-69. $\underline{U R L}$.

2. Wyszynski A, Wyszynski B. Manual de psiquiatría para pacientes con enfermedades médicas. 1a ed. Buenos Aires: Editorial Masson; 2006.

3. Monczor M. La indicación de psicofármacos en el embarazo y el posparto. En: Moizeszowicz J, editor. Psicofarmacología psicodinámica IV. Actualizaciones 2006. 1a ed. Buenos Aires: Paidós; 2006. p. 95-117. 
4. Wikinski S, Jufe G. El tratamiento farmacológico en psiquiatría: indicaciones, esquemas terapéuticos y elementos para su aplicación racional. 2a ed. Buenos Aires: Editorial Médica Panamericana; 2005.

5. Cohen LS. Treatment of bipolar disorder during pregnancy. J Clin Psychiatry. 2007; 68(9): 4-9. http://dx.doi.org/10.1017/S1092852900025712.

6. Appiani FJ. Psicofármacos, embarazo, lactancia: uso y seguridad. 1a ed. Buenos Aires: Jai Editorial; 2012.

7. Viguera AC, Whitfield T, Baldessarini RJ, Newport DJ, Stowe Z, Reminick A, et al. Risk of recurrence in women with bipolar disorder during pregnancy: prospective study of mood stabilizer discontinuation. Am J Psychiatry. 2007; 164: 1817-1824. http://dx.doi.org/10.1176/appi.ajp.2007.06101639.

8. Gómez-Elias CL. Manejo del trastorno bipolar en el embarazo. Rev Med MD. 2011; 3(3): 154-162. URL.

9. D'Ottone A. Tratamiento farmacológico del trastorno bipolar en el adulto. Rev Psiquiatr Urug. 2008; 72(1): 21-64. URL.

10. Gaviria SL. Tratamiento del trastorno afectivo bipolar en el embarazo. Rev Chil NeuroPsiquiat. 2008; 46(1): 43-54. http://dx.doi.org/10.4067/S0717-92272008000100006

11. Viguera AC, Cohen LS, Baldessarini RJ, Nonacs R. Managing bipolar disorder during pregnancy: weighing the risks and benefits. Can J Psychiatry. 2002; 47(5):426-436. http://dx.doi.org/10.1177/070674370204700503

12. Yonkers KA, Wisner KL, Stowe Z, Leibenluft E, Cohen L, Miller L, et al. Management of bipolar disorder during pregnancy and the postpartum period. Am J Psychiatry. 2004; 161(4): 608-620. http://dx.doi.org/10.1176/appi.ajp.161.4.608.

13. Correa Donoso E, Martínez JC, Ivanovic-Zuvic F, Uribe D. Enfermedad bipolar en la mujer en edad fértil. Rev CES Med. 2006; 20(2): 35-52. URL.

14. Medrano Albéniz J, Zardoya Mateo MJ, Pacheco Yáñez L, autores. Uso de psicofármacos en el embarazo y la lactancia. 1a ed. Badalona: Euromedice; 2009.

15. Lozano Suárez M, Ramos Brieva JA. Utilización de los psicofármacos en psiquiatría de enlace. 1a ed. Barcelona: Masson; 2002.

16. NICE: National Institute for Health and Care Excellence [Internet]. Londres: NICE; 17 dic 2014 [actualizado jun 2015; citado 7 sep 2016]. Antenatal and postnatal mental health: clinical management and service guidance. Disponible en: https://www.nice.org.uk/guidance/cg192 